\title{
Temporospatial clustering of idiopathic sudden onset low-tone sensorineural hearing loss: our experience with three patients
}

\author{
Vasileios Gkiousias $^{1}$, Sam Blakemore ${ }^{1}$, and Mahmood Bhutta ${ }^{2}$ \\ ${ }^{1}$ Royal Sussex County Hospital \\ ${ }^{2}$ Brighton and Sussex University Hospitals NHS Trust
}

May 26, 2020

\section{Main document}

\section{Key Points}

1. Sudden sensorineural hearing loss remains one of the most challenging conditions in modern otology

2. There is ambiguity in terms of its aetiology and management

3. Sudden sensorineural hearing loss is often idiopathic

4. We report temporospatial clustering of idiopathic sudden onset sensorineural hearing loss in three cases

5. Our findings add to the evidence that a transmissible agent such as a virus underlies aetiology in at least a proportion of such cases

\section{Introduction}

Sudden sensorineural hearing loss (SSNHL) is a rare emergency. Despite having been described first by De Kleyn in 1944 (1), SSNHL continues to remain one of the most challenging and controversial conditions in modern otology. It is defined as a hearing loss developing within three days, and confirmed by audiometric testing demonstrating a decrease of at least 30 decibels in hearing thresholds across three consecutive frequencies (comparing the affected to the opposite ear) (2). In practice, the diagnosis is often made with lesser degrees of hearing loss.

Insurance data from the USA suggests that SSNHL affects 27 per 100,000 individuals per annum (3). SSNHL is a disorder of uncertain aetiology, with only half of those affected recovering hearing (4) and many reporting persistent disability (5). It is often treated with systemic or local steroids, given within 14 days of symptom onset.

It is believed that SSNHL can result from autoimmune, metabolic, neurological, otological or vascular disease, infections, neoplasms, ototoxic agents, or trauma (4). However, in the majority of cases the cause is not evident. A number of ideas have been proposed to explain idiopathic SSNHL, and evidence from clinical, pathological, radiological and animal studies suggest that exposure to a virus (or one of a number of viruses) is the most likely aetiology in many idiopathic cases (4).

Here we present the first report of temporospatial clustering of three cases of idiopathic SSNHL, all presenting with unilateral low frequency loss. Such clustering suggests an environmental trigger to aetiology, consistent with a transmissible agent such as a virus.

\section{Patients and Methods}

\section{Patients}

The cases included one audiologist and two secretarial support staff to the audiology department, all working 
at a tertiary care hospital in South East England. The cases presented with SSNHL between March and July 2019.

\section{Methods}

Records of three patients, including medical notes, clinic letters, audiology reports, blood test and imaging results were examined retrospectively after the occurrence of SSNHL.

\section{Ethical considerations}

Informed consent was obtained by all three patients.

\section{Results}

Over the period under examination, three adult patient cases were reviewed.

The first patient was a 30-year-old lady who worked as an Audiologist in our hospital. She developed leftsided SSNHL on 5th March 2019, associated with ipsilateral sensation of aural fullness and distorted speech. She had a past medical history of migraines, for which she took prophylactic propranolol. She presented to medical services two days after symptom onset. Otoscopy was unremarkable, and a pure tone audiogram (PTA) showed a low frequency hearing loss in the left ear, on a background of mild bilateral high frequency hearing loss (Figure 1). The high frequency hearing loss had been documented on a PTA a year earlier and was unchanged. She was prescribed a week of oral steroids and subsequent review showed complete recovery (Figure 1). Two months later the patient experienced a recurrence of left-sided SSNHL, which also resolved following a week of oral steroids. In view of the recurrent episodes, further investigations were arranged after symptom resolution. These results were unremarkable, including serum thyroid function, haematology, erythrocyte sedimentation rate, anti-neutrophil cytoplasmic antibody levels, and Lyme disease serology, and normal MRI of the internal auditory meati.

The second patient was a 61-year-old lady who worked as secretarial support for the audiology department. She developed right-sided SSNHL on 13th May 2019 without associated symptoms. She had a past medical history of Type 1 diabetes mellitus. The patient presented to medical services a day after the onset of her symptoms. Otoscopy was normal. PTA showed low frequency sensorineural hearing loss on the right side and normal hearing on the left (Figure 2). The decision was made to proceed with intratympanic rather than oral steroid therapy so that serum glucose control would not be perturbed. Four intratympanic injections were initially scheduled, each 2-3 days apart, but were discontinued after the second injection due to complete symptom resolution and return of hearing to normal (Figure 2). No subsequent investigations took place and there have not been any further symptoms.

The third patient was a 24-year-old man who also worked as secretarial support for the audiology department. He developed right-sided SSNHL a couple in late March 2019. but did not present to clinic until 18th July 2019. He did not seek medical advice at the time of symptom onset, as he attributed his hearing loss to wax accumulation. He had no associated otological symptoms, and there was no past medical, surgical or medication history of note, however his mother had worn bilateral bone-anchored hearing aids. Otoscopy was normal. PTA demonstrated low frequency sensorineural hearing loss on the right side, with normal hearing on the left (Figure 3). In view of his delayed presentation, he was not offered oral steroids but was investigated with MRI, which showed normal internal auditory meati. There has been no resolution of hearing in this patient to date (Figure 3).

There have been no further cases in our audiology department since July 2019.

\section{Probability of clustering}

We calculated the probability of clustering if these three cases were independent events. Age standardised incidence rates from the USA (3) suggest the annual incidence of SSNHL in a 30-year-old (first case) is 12 per 100,000; for a 61-year-old (second case) is 47 per 100,000 and for a 24-year-old (third case) is 12 per 100,000. Discounting the index case, the combined per-person probability of a second and third case occurring in the 
space of a year, multiplied by the 28 regular staff working in the audiology department at the time, gives an overall probability of:

$47 / 100,000 * 12 / 100,000 * 28=1.6 \times 10^{-6}$

Therefore, it is highly unlikely that these are independent events.

\section{Discussion}

Our report describes temporospatial clustering of SSNHL in an audiology department in South East England.

The temporospatial clustering of these cases suggests they are caused by a common environmental agent. Clinical experience is that chemical or other environmental compounds tend to cause bilateral hearing loss, whereas viruses may cause unilateral hearing loss. The parsimonious (but unproven) explanation for the clustering we see here is that of a transmissible virus spread between cases.

What makes a single aetiological agent even more likely is the near identical pathology in all three cases (Figures 1-3), of an upsloping low frequency sensorineural hearing loss. In general, low frequency SSNHL is associated with higher rates of recovery (4) compared to high frequency loss, and in some cases may be a prelude to the onset of Ménière's disease. However, those who go on to develop Ménière's disease in subsequent years tend to present with additional symptoms of tinnitus or vertigo (7), which were not found in these three cases.

Viral infection is thought to underlie other sudden inner ear disorders, including vestibular neuritis, labyrinthitis, and idiopathic (Bell's) facial palsy. In Bell's palsy occupational clustering has also been reported (8) (9), albeit without identification of an aetiological agent. The pathology underlying the hearing loss in the cases presented here can only be conjecture, however the low frequency loss of the audiogram suggests the virus (or other aetiological agent) is either causing cochlear hydrops or is preferentially affecting hair cells or neurons of the cochlear apex.

In 2019, Gilani and Shin hypothesised there may be temporospatial clustering of SSNHL, but their visual inspection of data on attendances for SSNHL at a single tertiary referral centre in the United States did not provide supporting evidence (6). However, retrospective analysis of aggregate data from a single institution will likely fail to identify clustering if those with SSNHL do not present at the same time or location. This could be for example because of a delay in onset between cases, because those affected do not present to medical services at the same time (or at all), or because they present to primary or emergency care, or to different providers of specialist care. It is also possible that clustering only happens in some subtypes of SSNHL (such as low frequency loss), which will again be difficult to identify from aggregate data. All the cases presented here were employed in the same audiology department, and so they presented for assessment at their place of employment, making recognition of clustering easy.

To the best of our knowledge, this is the first report of temporospatial clustering of idiopathic SSNHL. Our findings add to the evidence that a transmissible agent such as a virus underlies aetiology in at least a proportion of such cases.

\section{References}

1. Rennie C, Selvadural DK. Sudden sensorineural hearing loss-aetiology and management. J ENT Mastercl. $2008 ; 45-50$.

2. Chandrasekhar SS, Tsai Do BS, Schwartz SR, Bontempo LJ, Faucett EA, Finestone SA, et al. Clinical practice guideline: sudden hearing loss (update). Otolaryngol - Head Neck Surg (United States). 2019;161(1_suppl):S1-45.

3. Alexander TH, Harris JP. Incidence of sudden sensorineural hearing loss. Otol Neurotol. 2013;34(9):1586-9.

4. Kuhn M, Heman-Ackah SE, Shaikh JA, Roehm PC. Sudden sensorineural hearing loss: a review of diagnosis, treatment, and prognosis. Trends Amplif. 2011;15(3):91-105. 
5. Chiossoine-Kerdel JA, Baguley DM, Stoddart RL, Moffat DA. An investigation of the audiologic handicap associated with unilateral sudden sensorineural hearing loss. Am J Otol. 2000;21(5):645-51.

6. Gilani S, Shin JJ. Time and geographic clustering of adult patients with unilateral idiopathic sudden sensorineural hearing loss. Med Hypotheses. 2019;122(July 2018):184-7.

7. Stölzel K, Droste J, Voß LJ, Olze H, Szczepek AJ. Comorbid symptoms occurring during acute low-tone hearing loss (AHLH) as potential predictors of Menière's disease. Front Neurol. 2018;9:1-6.

8. Frumkin H, Horowitz M, Jabre JF, Payton M, Kantrowitz W. An investigation of a workplace cluster of bell's palsy. Vol. 34, Journal of Occupational Medicine. 1992. p. 1064-70.

9. Reaves EJ, Ramos M, Bausch DG. Workplace cluster of Bell's palsy in Lima, Peru. BMC Res Notes. 2014;7(1):1-5.

\section{Hosted file}

Figure 1.docx available at https://authorea.com/users/325837/articles/453858-temporospatialclustering-of-idiopathic-sudden-onset-low-tone-sensorineural-hearing-loss-our-

experience-with-three-patients

\section{Hosted file}

Figure 2.docx available at https://authorea.com/users/325837/articles/453858-temporospatialclustering-of-idiopathic-sudden-onset-low-tone-sensorineural-hearing-loss-ourexperience-with-three-patients

\section{Hosted file}

Figure 3.docx available at https://authorea.com/users/325837/articles/453858-temporospatialclustering-of-idiopathic-sudden-onset-low-tone-sensorineural-hearing-loss-ourexperience-with-three-patients 